\title{
Diagnostic accuracy of refractometry for assessing bovine colostrum quality: A systematic review and meta-analysis
}

\author{
S. Buczinski ${ }^{* 1}$ and J. M. Vandeweerd $†$ \\ *Département des Sciences Cliniques, Faculté de Médecine Vétérinaire, Université de Montréal, CP 5000, St-Hyacinthe, J2S 7C6, Québec, \\ Canada \\ †Integrated Veterinary Research Unit, Namur Research Institute for Life Sciences, Department of Veterinary Medicine, Faculty of Sciences, \\ Université de Namur, Rue de Bruxelles 61, 5000 Namur, Belgium
}

\section{ABSTRACT}

Provision of good quality colostrum [i.e., immunoglobulin $\mathrm{G}$ ( $\mathrm{IgG}$ ) concentration $\geq 50 \mathrm{~g} / \mathrm{L}$ ] is the first step toward ensuring proper passive transfer of immunity for young calves. Precise quantification of colostrum IgG levels cannot be easily performed on the farm. Assessment of the refractive index using a Brix scale with a refractometer has been described as being highly correlated with $\operatorname{IgG}$ concentration in colostrum. The aim of this study was to perform a systematic review of the diagnostic accuracy of Brix refractometry to diagnose good quality colostrum. From 101 references initially obtained, 11 were included in the systematic review meta-analysis representing 4,251 colostrum samples. The prevalence of good colostrum samples with IgG $\geq 50$ $\mathrm{g} / \mathrm{L}$ varied from 67.3 to $92.3 \%$ (median $77.9 \%$ ). Specific estimates of accuracy [sensitivity (Se) and specificity (Sp)] were obtained for different reported cut-points using a hierarchical summary receiver operating characteristic curve model. For the cut-point of $22 \%$ ( $\mathrm{n}=$ 8 studies), $\mathrm{Se}=80.2 \%$ (95\% CI: $71.1-87.0 \%)$ and $\mathrm{Sp}$ $=82.6 \%(71.4-90.0 \%)$. Decreasing the cut-point to $18 \%$ increased Se $[96.1 \%(91.8-98.2 \%)]$ and decreased Sp [54.5\% (26.9-79.6\%)]. Modeling the effect of these Brix accuracy estimates using a stochastic simulation and Bayes theorem showed that a positive result with the $22 \%$ Brix cut-point can be used to diagnose good quality colostrum (posttest probability of a good colostrum: $94.3 \%$ (90.7-96.9\%). The posttest probability of good colostrum with a Brix value $<18 \%$ was only $22.7 \%(12.3-39.2 \%)$. Based on this study, the 2 cutpoints could be alternatively used to select good quality colostrum (sample with Brix $\geq 22 \%$ ) or to discard poor quality colostrum (sample with Brix $<18 \%$ ). When sample results are between these 2 values, colostrum supplementation should be considered.

Received January 27, 2016.

Accepted May 27, 2016.

${ }^{1}$ Corresponding author: s.buczinski@umontreal.ca
Key words: immunoglobulin G, sensitivity, specificity, accuracy, Brix

\section{INTRODUCTION}

Colostrum is the first mammary secretion composed of various components including immunoglobulins, which are actively secreted during the last weeks of pregnancy in mammals (Baumrucker et al., 2010). In bovine species, colostrum is critical because calves are born agammaglobulinemic. The key immunity component of colostrum is IgG, although white blood cells and cytokines are also important for calf immunity (Stelwagen et al., 2009). Gut permeability to IgG absorption decreases rapidly after birth (Baumrucker and Bruckmaier, 2014). Delivering good quality colostrum (IgG $\geq 50 \mathrm{~g} / \mathrm{L}$ ) as quickly as possible after birth is a critical component to calf health programs. It is essential to optimize transfer of maternal immunity when the gastrointestinal tract can absorb antibodies (Godden, 2008). Colostrum IgG content is one important component for achieving good passive transfer of immunity to calves. Other factors such as colostrum contamination (Gelsinger et al., 2015), quantity and time at first colostrum feeding (Stott et al., 1979; Osaka et al., 2014) as well as other specific calves characteristics that may have an effect on absorption capacity (Tyler and Ramsey, 1991; Sangild, 2003) should also be taken into account when establishing colostrum feeding protocols.

The quality of colostrum can vary widely in terms of IgG concentration. In a recent US study on 827 samples in 67 herds, almost $30 \%$ of colostrum samples had an IgG concentration $<50 \mathrm{~g} / \mathrm{L}$ (Morrill et al., 2012a). This arbitrary cut-point was based on the fact that calves need to receive 100 to $200 \mathrm{~g}$ of $\mathrm{IgG}$ to improve the chances of achieving success in passive transfer immunity (defined as serum $\operatorname{IgG} \geq 10 \mathrm{~g} / \mathrm{L}$ obtained 1 to $7 \mathrm{~d}$ after birth) with 3 to $4 \mathrm{~L}$ of colostrum fed within $6 \mathrm{~h}$ after birth (McGuirk and Collins, 2004). This cut-point has been widely used to define good and poor quality colostrum and reported in different original articles or 
reviews as an industry standard (Chigerwe et al., 2008; Godden, 2008; Chigerwe and Hagey, 2014). The IgG concentration of colostrum can be assessed by several methods. The most commonly reported method is radial immunodiffusion assay (RID), which is considered the gold standard method (Fleenor and Stott, 1981). Immunoglobulin $\mathrm{G}$ concentrations obtained by nearinfrared spectrometry (NIRS) have been reported to be highly correlated with IgG concentration measured by RID $\left(r^{2}=0.95\right.$; Rivero et al., 2012) and can therefore also be considered as another gold standard test. The major drawback of these methods is that they are not practical on farms (Rivero et al., 2012; Bartier et al., 2015).

On-farm methods for assessing colostrum quality include color and specific gravity (Gross et al., 2014; Bartier et al., 2015). However, they are not widely used in Canada, as shown in a recent survey 115 dairy farms from the province of Québec, where none of the farms assessed colostrum quality (Vasseur et al., 2010). Reasons for not using these methods could be variable. Lack of accuracy of color assessment has been reported with low sensitivity (Se) and specificity (Sp) of $50 \%$ (Gross et al., 2014). Other limitations are the effect of sample temperature on accuracy as well as fragility of the colostrometer and the necessity to clean it between analyses.

More recently, the refractive index converted on a Brix scale using optical or digital handheld devices has been mentioned as a valuable indicator to distinguish good quality (IgG $\geq 50 \mathrm{~g} / \mathrm{L}$ ) versus poor quality ( $\operatorname{IgG}$ $<50 \mathrm{~g} / \mathrm{L}$ ) colostrum (Bielmann et al., 2010; Quigley et al., 2013; Bartier et al., 2015). Using Brix refractometry could be easily implemented on farms since only a few drops of colostrum are required and the result is immediately known. Similar devices have been validated to monitor transfer of passive immunity in dairy calves (Deelen et al., 2014).

The Brix scale refers to the sugar content of a solution. One Brix percentage corresponds to $1 \mathrm{~g}$ of sucrose diluted in $100 \mathrm{~g}$ of a solution (Son et al., 2009). The Brix refractometer can be optical or digital, the latter being more appealing since it gives a rapid objective measurement of the Brix percent of the sample. Various Brix values with various sensitivities (Se: proportion of colostrum above the Brix cut-point that is truly of good quality) and specificities (Sp: proportion of colostrum below the Brix cut-point that is truly of poor quality) have been reported (Bielmann et al., 2010; Bartier et al., 2015; Morrill et al., 2015). The discrepancy between studies may be due either to intra-study variability (related to sample size and study variance, therefore precision of Se and Sp estimates) or inter-study vari- ability (due to the differences observed in the different study estimates; Macaskill et al., 2010). Systematic review methods have been developed as a practical way to summarize all the information to answer to a specific question in various research disciplines including animal agriculture and veterinary medicine (Sargeant and O'Connor, 2014). This evidence-based approach can also be applied for summarizing scientific quality (i.e., study methods) and quantitative information (i.e., Se and Sp using meta-analytic methods) on diagnostic test accuracy (Leeflang, 2014). Summarizing information about the use of Brix refractometer to assess colostrum quality would therefore be useful for encouraging its use in dairy farms.

The objective of the current study was to perform a systematic review and meta-analysis of the accuracy of the index test Brix to select good quality colostrum in cattle (defined as an IgG concentration $\geq 50 \mathrm{~g} / \mathrm{L}$ identified by the reference standards RID or NIRS). The second objective of the study was to summarize the accuracy of specific Brix cut-points to select good quality colostrum.

\section{MATERIALS AND METHODS}

\section{Literature Search and Selection Criteria}

A systematic search of the literature was performed from January 1973 to October 2015 on PubMed, CAB Abstract, and searchable proceedings of animal conferences (S-PAC) to retrieve all pertinent trials reporting the accuracy of the index test (Brix refractometer) versus the reference standard (IgG determination by RID or NIRS). The search strategy was based on the keywords: (Brix or refractometer or refractometry) AND (colostrum or colostral) AND (cow or cattle or ox or bovine). The language filter was restricted to papers written in English, French, German, and Spanish. The reference list of each selected article was further screened for any relevant article that was not identified by the initial search strategy (gray literature). Another search was performed using Google scholar with the research keywords: "Brix and colostrum." We did not filter the search in terms of cow breed because we hypothesized that breed characteristics would only affect prevalence of good quality colostrum (Conneely et al., 2013) and not affect test accuracy.

The titles were screened for their pertinence to the question and then the final databases were merged together to exclude duplicates. The scientific papers identified by Google Scholar and the S-PAC were added to the list of eligible publications. The articles and abstracts were then fully read and included in the 
review if they actually compared Brix to RID or NIRS. The references were then imported into freely available software [Review Manager (RevMan), Version 5.3, Copenhagen: The Nordic Cochrane Centre, The Cochrane Collaboration, 2014]. The studies were selected for further analysis if they assessed the accuracy of cutpoint values for optical or digital Brix refractometry by comparison to the IgG concentration measured by a gold standard technique (RID or NIRS) and if the cut-point values of $\operatorname{IgG} \geq 50$ and $<50 \mathrm{~g} / \mathrm{L}$ were the criteria used to define colostrum of good and poor quality, respectively.

Finally, studies were retained only if a $2 \times 2$ table (for at least one Brix cut-point vs. the reference standard) could be retrieved in the publication either directly, or indirectly from elements necessary to create such a table [total number of samples, Se, Sp, prevalence of good quality colostrum (p), positive or negative predictive values]. For studies presenting multiple cut-points or multiple Brix refractometer results, all possible $2 \times$ 2 tables were collected.

\section{Internal and External Validity}

To assess the internal validity of each retained study (i.e., risk of bias), as well as the external validity of the study to the review question (i.e., applicability), the quality of diagnostic accuracy studies (QUADAS-2) tool was used (Whiting et al., 2011).

The QUADAS-2 was used to assess the risk of bias in 4 specific study domains including: (1) patient selection (e.g., whether a consecutive or random selection of samples was performed), or whether exclusion of a subgroup of samples was performed where the index test could potentially give different results (e.g., excluding blood-tinted colostrum), or whether cohort or casecontrol studies were used (e.g., case-control designs classically overestimate test accuracy; Rutjes et al., 2005), (2) the use of the test under review (i.e., if Brix refractometry was performed with operators blind to reference standard results and if the accuracy threshold was prespecified or obtained in a data-driven way), (3) the use of the reference test (i.e., if RID or NIRS were likely to classify good versus poor colostrum as well as if their results were obtained without any knowledge about using the Brix test), and (4) the flow and timing of patient testing and status determination (e.g., whether there was a delay between index and reference tests).

The QUADAS-2 was also used to assess external validity in 3 applicability domains concerning how well the review question was matched with (1) patient selection (were study populations and samples truly adequate to answer the question?), (2) index test (was the method of conducting the Brix test or interpretation really applicable?), and (3) reference standards [was the definition (by RID and NIRS) of good and poor colostrum adequate?: e.g., the results of a study which used a homemade RID technique versus a commercially validated technique could not be considered to be equally applicable].

Even if no specific score was attributed to the papers, this tool has been described as an efficient method to assess the relative strengths and weaknesses of diagnostic accuracy studies included in a systematic review process. The QUADAS-2 has been standardly used in human medicine for systematic reviews of diagnostic test accuracy (Leeflang et al., 2013).

\section{Statistical Analysis}

The statistical analysis was performed according to the recommendations of the Cochrane diagnostic test accuracy group (Macaskill et al., 2010) using 2 different software programs (RevMan 5.3 and SAS version 9.3, SAS Institute Inc., Cary, NC). At the study level, all of the specific information was collected on a spreadsheet (Excel, Microsoft, Redmond, WA). This included type of publication (peer-reviewed vs. non-peer-reviewed), language of publication, country, prevalence of goodquality colostrum, characteristics of study population (breed, parity), mean IgG concentration with its variations (SD or ranges), as well as the type of handling of the sample before Brix reading (fresh, refrigerated, or frozen).

The $2 \times 2$ tables obtained from each study were used to perform meta-analytic models. The tables were either directly collected from study results, or constructed on the basis of reported Se, Sp, prevalence of good colostrum defined as the proportion of colostrum with a concentration of $\mathrm{IgG} \geq 50 \mathrm{~g} / \mathrm{L}(p)$, and total number of samples $(n)$. Different equations were used to determine the $2 \times 2$ table cells:

(i) true positive cases (TP: cases with an index test positive (i.e., $\geq$ Brix cut-point) and colostrum $\operatorname{IgG} \geq 50 \mathrm{~g} / \mathrm{L})=$ Se $\times p \times n$;

(ii) true negative cases (TN: $<$ Brix cut-point and colostrum $\operatorname{IgG}<50 \mathrm{~g} / \mathrm{L})=\operatorname{Sp} \times(1-p) \times n$;

(iii) false positive cases (FP: $\geq$ Brix cut-point and colostrum $\operatorname{IgG}<50 \mathrm{~g} / \mathrm{L})=(1-\mathrm{Sp}) \times(1-p)$ $\times n$;

(iv) false negative cases (FN: $<$ Brix cut-point and colostrum $\operatorname{IgG} \geq 50 \mathrm{~g} / \mathrm{L})=(1-\mathrm{Se}) \times p \times n$.

In studies reporting $\mathrm{Se}, \mathrm{Sp}$, and predictive values of the test (positive and negative predictive values), the 
prevalence of good quality colostrum $(p)$ was obtained using the formula

$$
p=(S p-1) /\left(S e+S p-1-\left(\frac{S e}{P P V}\right)\right) .
$$

The corresponding $2 \times 2$ tables were then derived to calculate TP, TN, FP, and FN.

\section{Accuracy of Brix Refractometer for All Possible Cut-Points}

The $2 \times 2$ tables for the main Brix cut-point values proposed in previous studies were retrieved for the main analysis. In studies reporting more than one cut-point, or not mentioning a specific value, $2 \times 2$ tables for a specific cut-point were randomly chosen among the different reported cut-points.

The corresponding $2 \times 2$ tables were then used to obtain the hierarchical summary receiver operating characteristic (HSROC) curve using the NLMIXED procedure with SAS (version 9.3, SAS Institute Inc.) and the METADAS macro (Rutter and Gatsonis, 2001; Takwoingi and Deeks, 2008). The HSROC model was used to determine global test accuracy taking into account the fact that different thresholds could be reported in a study.

The HSROC model takes into account the within study variation (1) and between study variation (2) using the following model:

$$
\operatorname{Logit}\left(\pi_{i j}\right)=\left(\theta_{i}+\alpha_{i} \mathrm{X}_{i j}\right) \times \mathrm{e}^{-\beta \mathrm{X} i j} .
$$

The model describes, on the logit scale, the probability of a positive test $\left(\pi_{i j}\right)$ of a sample in the study $i$ with a quality $j\left\{j=0\right.$ for samples with $\operatorname{IgG}<50 \mathrm{~g} / \mathrm{L}\left[\pi_{i 0}=\right.$ false positive fraction $(\mathbf{F P F}=1-\mathrm{Sp})$ in study $i]$ and $j=1$ for samples with $\operatorname{IgG} \geq 50 \mathrm{~g} / \mathrm{L}\left[\pi_{i 1}=\right.$ true positive fraction $(\mathbf{T P F}=$ Se) in study $i]\}$. The variable $\mathrm{X}_{i j}$ denotes the true colostrum quality for a sample in the study $i$ with a quality $j$. The parameters $\theta_{i}$ (cut-point parameter) and $\alpha_{i}$ (accuracy parameter $=\mathrm{TPF}-\mathrm{FPF}$ ) may vary between studies. The last parameter $(\beta)$ is the scale or shape parameter that allows for modeling of receiver operating characteristic curve asymmetry. The between study variation of $\theta$ and $\alpha$ can be assumed to have independent normal distribution that could also be influenced by any covariate at the study level (Takwoingi and Deeks, 2008; Macaskill et al., 2010).

\section{Sensitivity Analysis}

Sensitivity analysis was used to look at the effect of decisions made during the review process of the sys- tematic review findings (Macaskill et al., 2010). It was not a statistical analysis but it assessed whether or not review findings were based on arbitrary decisions made by the authors. A sensitivity analysis was therefore performed with successive removal of studies using NIRS as reference standard test and studies of non-Holstein cows $(\mathrm{n}=2)$. The 2 summary ROC curves were obtained including and excluding the studies with the factor of interest (i.e., studies using NIRS and studies using non-Holstein cows) using default RevMan graphs, and visual comparisons of summary receiver operating characteristic (sROC) curve were made (Macaskill et al., 2010).

\section{Subgroup Analysis}

A subgroup analysis was performed to compare the effect of a specific study covariate on Brix accuracy (Macaskill et al., 2010). The covariate that was anticipated to potentially have an effect on test accuracy was whether or not the study was peer-reviewed (Macaskill et al., 2010; van Enst et al., 2014). Case-control (2-gate) versus cohort (1-gate) studies (Rutjes et al., 2005) and digital versus optical refractometry were also initially considered as covariates but were not further explored due to the lack of case-control studies and the variability of refractometers used (different optical and digital refractometers).

\section{Accuracy of Specific Brix Cut-Points}

The specific Brix cut-points of 18\% (Silva, 2013; Doussoulin, 2014; Morrill et al., 2015), 21\% (Quigley et al., 2013), and 22\% (Bielmann et al., 2010; Arnholdt et al., 2014; Chamorro et al., 2015) have been proposed in the literature to distinguish poor quality from good quality colostrum. We performed secondary analyses for these specific prespecified cut-points, using studies where corresponding $2 \times 2$ tables were available with the HSROC model. A specific point estimate of Se/ Sp 95\% CI as well as the positive and negative likelihood ratio, were determined for each studied cut-point (Takwoingi and Deeks, 2008).

\section{Publication Bias}

The publication bias assessment considers the probability that studies of smaller size but large effect (i.e., apparently higher accuracy, due to within study variability) are more frequently reported than studies without a large effect (i.e., studies showing lower accuracy). The publication bias assessment represents specific challenges in diagnostic accuracy meta-analysis due to the bivariate nature of these studies and the fact that 
no formal hypothesis testing concerning test accuracy is reported, in contrast to intervention studies. The Se and $\mathrm{Sp}$ (i.e., the 2 reported accuracy measures) are determined in 2 distinct populations (Se in the colostrum with $\operatorname{IgG} \geq 50 \mathrm{~g} / \mathrm{L}$ and $\mathrm{Sp}$ in colostrum with $\mathrm{IgG}<50$ $\mathrm{g} / \mathrm{L}$ ) that have inherently different sample sizes due to the study design (case control vs cohort) or prevalence of the diagnosed event (i.e., prevalence of good quality colostrum).

The Deeks test was reported to take into account these specific challenges (Deeks et al., 2005) and is the most robust test for reporting publication bias in systematic reviews of diagnostic tests (van Enst et al., 2014). It was therefore used for detecting publication bias in this study using the same data set as the one for the Brix accuracy across the different cut-points (Deeks et al., 2005). This test is based on reporting the association between the natural logarithm of the diagnostic odds ratio $[\mathrm{DOR}=(\mathrm{TP} \times \mathrm{TN}) /(\mathrm{FP}+\mathrm{FN})]$ and $1 /(\mathrm{ESS})^{1 / 2}$, where ESS is the effective sample size defined as a function of the number of cases $(\mathrm{TP}+\mathrm{FN})$ and number of noncases $(\mathrm{TN}+\mathrm{FP})$ as follows:

$$
\begin{aligned}
\mathrm{ESS}= & 4 \times(\mathrm{TP}+\mathrm{FN}) \times(\mathrm{TN}+\mathrm{FP}) / \\
& (\mathrm{TP}+\mathrm{FN}+\mathrm{TN}+\mathrm{FP}) .
\end{aligned}
$$

\section{Practical Implication of Brix Accuracy}

The practical application of the meta-analysis results were determined using the $\mathrm{Se} / \mathrm{Sp}$ estimates (mode and 95\% CI) obtained from 18, 21, and 22\% Brix specific cut-points. These 3 estimates (mean, 2.5th and 97.5th percentile) for both cut-points were used to create a specific PERT distribution for Se, Sp, and the prevalence of good quality colostrum (using the median, minimum, and maximum values of $p$, which were determined as the $\left.p_{\text {pretest }}\right)$ in the studies included in the systematic review. The PERT distribution has been commonly used for modeling expert opinion defined by only 3 parameters (a minimum, maximum, and most probable value) in stochastic modeling. This distribution has interesting modeling properties when compared with other nonparametric distribution such as triangle distribution. The PERT distribution is less influenced by the minimum and the maximum values than a triangular distribution. This property takes into account the fact that (most of the time) the most probable value is generally better defined than its ranges (Lehman et al., 2011). The 3 parameters (Se, Sp, and $p_{\text {pretest }}$ ) were used for determining posttest probability ( $p_{\text {posttest }}$ ) of good quality colostrum based on Bayes theorem (Caraguel and Vanderstichel, 2013):
$\mathrm{LR} \times p_{\text {pretest }} /\left(1-p_{\text {pretest }}\right)=p_{\text {posttest }} /\left(1-p_{\text {posttest }}\right)$.

A stochastic modeling approach was then used to obtain posttest probability of good quality colostrum based on a positive (using the positive likelihood ratio of the test) or negative (using the negative likelihood ratio of the test) Brix result. The posttest probability $\left(p_{\text {posttest }}\right)$ densities were obtained using a Monte-Carlo simulation with 50,000 iterations (ModelRisk, Vose Software BVBA, Ghent, Belgium).

\section{RESULTS}

The flow diagram summarizing the research strategy is depicted in Figure 1. A total of 11 studies were included in the systematic review. This represents 4,251 colostrum samples. Nine of 11 studies used RID as reference standard (Chigerwe et al., 2008; Bielmann et al., 2010; Morrill et al., 2012b, 2015; Quigley et al., 2013; Arnholdt et al., 2014; Vandeputte et al., 2014; Bartier et al., 2015; Chamorro et al., 2015), and 2 of them used NIRS for IgG concentration determination (Silva, 2013; Doussoulin, 2014). The prevalence of good quality colostrum (IgG concentration $\geq 50 \mathrm{~g} / \mathrm{L}$ ) varied from 67.3 to $92.3 \%$ (median $77.9 \%$, mean $78.8 \%$ ). The main characteristics of the studies are summarized in Table 1.

The overall quality of reporting of these studies is summarized in Figures 2A and 2B. The overall quality of the articles was good. Most of the unclear risks came from unspecified sampling strategies (i.e., absence of clear description of whether sampling was performed on consecutive calvings or not) as well as difficulties in determining if the index test was interpreted without knowledge of reference standard results (i.e., it was not explicitly stated in many studies if the researchers were blind to the first test result when performing the second test).

The accuracy in terms of Se and Sp for the specific studies (using the cut-points proposed by the authors) are summarized in Figure 3. The Se varied from 65 to $95 \%$ and Sp varied from 66 to $95 \%$. The HSROC curve obtained from all the available studies is summarized in Figure 4. There was more heterogeneity for Sp than for Se as shown in both figures. The precision of $\mathrm{Sp}$ estimates (to diagnose poor quality colostrum) was lower (wider 95\% CI) than for Se. The Deeks test did not reveal any publication bias based on the absence of a linear relation between $\operatorname{lnDOR}$ and $1 / \sqrt{ } \operatorname{ESS}(P=$ 0.48 ; Figure 5).

The sensitivity analysis is graphically presented in Figures $6 \mathrm{~A}$ and $6 \mathrm{~B}$. The sROC curves did not change 

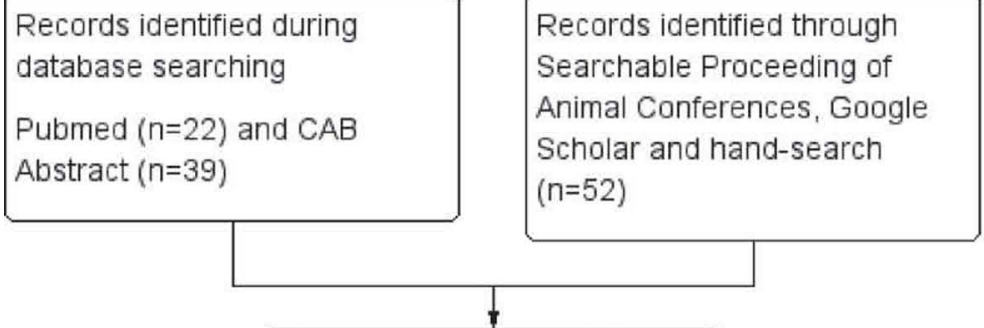

Records after duplicates removed $(n=101)$

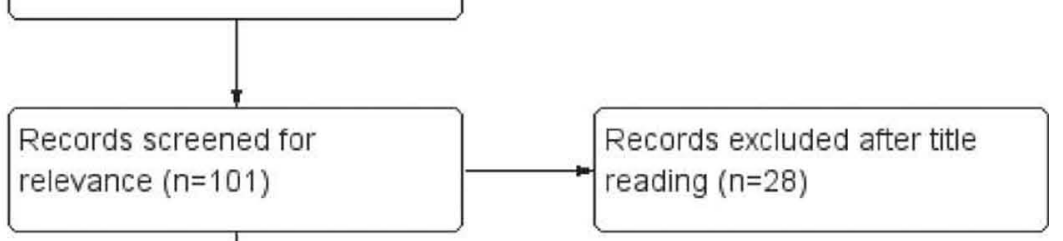

Records excluded $(n=57)$

Blood or serum IgG $(n=25)$

No Brix assessment $(n=10)$

No adequate reference standard $(n=5)$

Other colostrum characteristics $(n=7)$

Review $(n=6)$

Abstract from a published study $(n=4)$

Records abstracts screened for relevance $(n=73)$

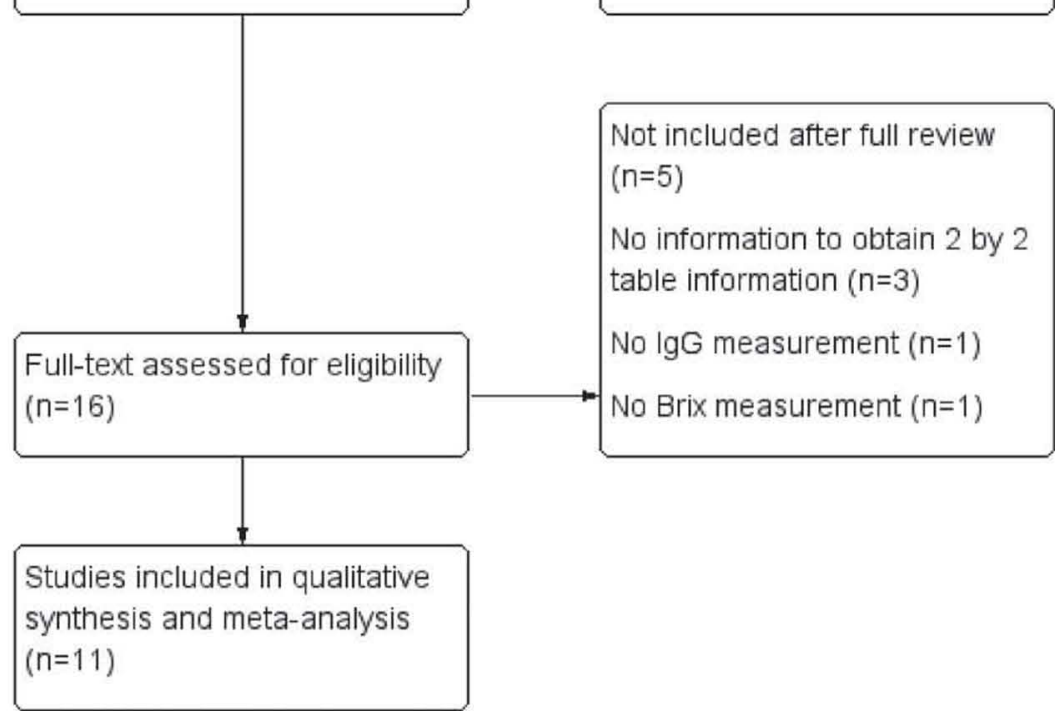

Figure 1. Flow diagram of the systematic review on the diagnostic ability of Brix refractometry to assess colostrum quality in cows. 


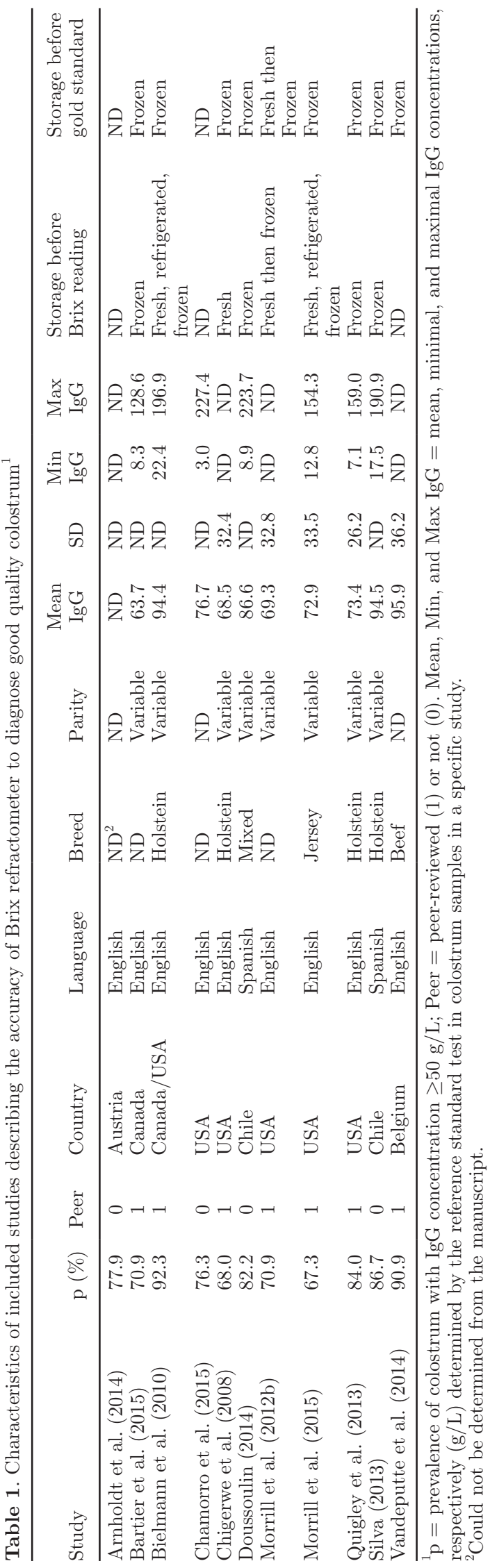

after removing studies that used NIRS as a reference standard to quantify IgG; this demonstrated the absence of an effect of the reference standard on Brix accuracy. There was a slight decrease in accuracy when removing these studies and those that used beef cattle (Vandeputte et al., 2014) or Jersey cows (Morrill et al., 2015) with a very small difference in the shape of the 2 SROC curves. However, it was not possible to determine whether this effect was statistically significant; it was visually estimated as low possibly because of the low number of remaining studies (7 vs. 11) for estimating sROC characteristics.

There was no difference in HSROC curves of studies that were or were not peer-reviewed $(P=0.42$ and 0.63 for relative Se and Sp, respectively), showing that this covariate was not associated with different Brix accuracy characteristics.

The summary estimates of all prespecified cut-points are summarized in Table 2. The sensitivity decreased when increasing the cut-point and this was accompanied by a better specificity. The HSROC curve, summary estimates, and 95\% CI when using the $22 \%$ Brix as a cut-point are depicted in Figure 7.

The practical implication of the Brix test was determined for the Brix cut-point of 22, 21, and $18 \%$ using a Monte Carlo simulation (Table 2). For a sample with a Brix $\geq 22 \%$, the posttest probability of having good quality (i.e., $\operatorname{IgG} \geq 50 \mathrm{~g} / \mathrm{L}$ ) colostrum was $94.3 \%$ (5th to 95 th percentile: $90.7-96.9 \%)$. Using this cut-point for defining good quality colostrum seemed to be a reasonable practice with, on average, less than $10 \%$ of misclassifications in herds with average colostrum quality comparable to the included studies. This probability decreased to $47.2 \%$ (5th-95th percentile: $35.5-62.3 \%$ ) when the Brix result was $<22 \%$, indicating that close to $50 \%$ of colostrum $<22 \%$ Brix still had a true $\operatorname{IgG}$ content $\geq 50 \mathrm{~g} / \mathrm{L}$ (Figure 8 ). Using $<18 \%$ as the cutpoint to detect poor quality colostrum (colostrum with IgG content $<50 \mathrm{~g} / \mathrm{L}$ ) seemed to be a better choice with only $22.7 \%$ misclassified colostrum.

\section{DISCUSSION}

To the authors' knowledge, this is the first systematic review reporting the diagnostic accuracy of the Brix refractometer to detect good quality colostrum. We found 11 studies reporting the accuracy of at least one Brix cut-point with NIRS or RID reference standard. This number was large enough to generate accuracy estimates of the Brix test. However, this number was too low to specifically analyze prespecified covariates that could potentially be associated with different test accuracy (e.g., breed or reference standard used). The choice of the reference standard is a critical issue when 


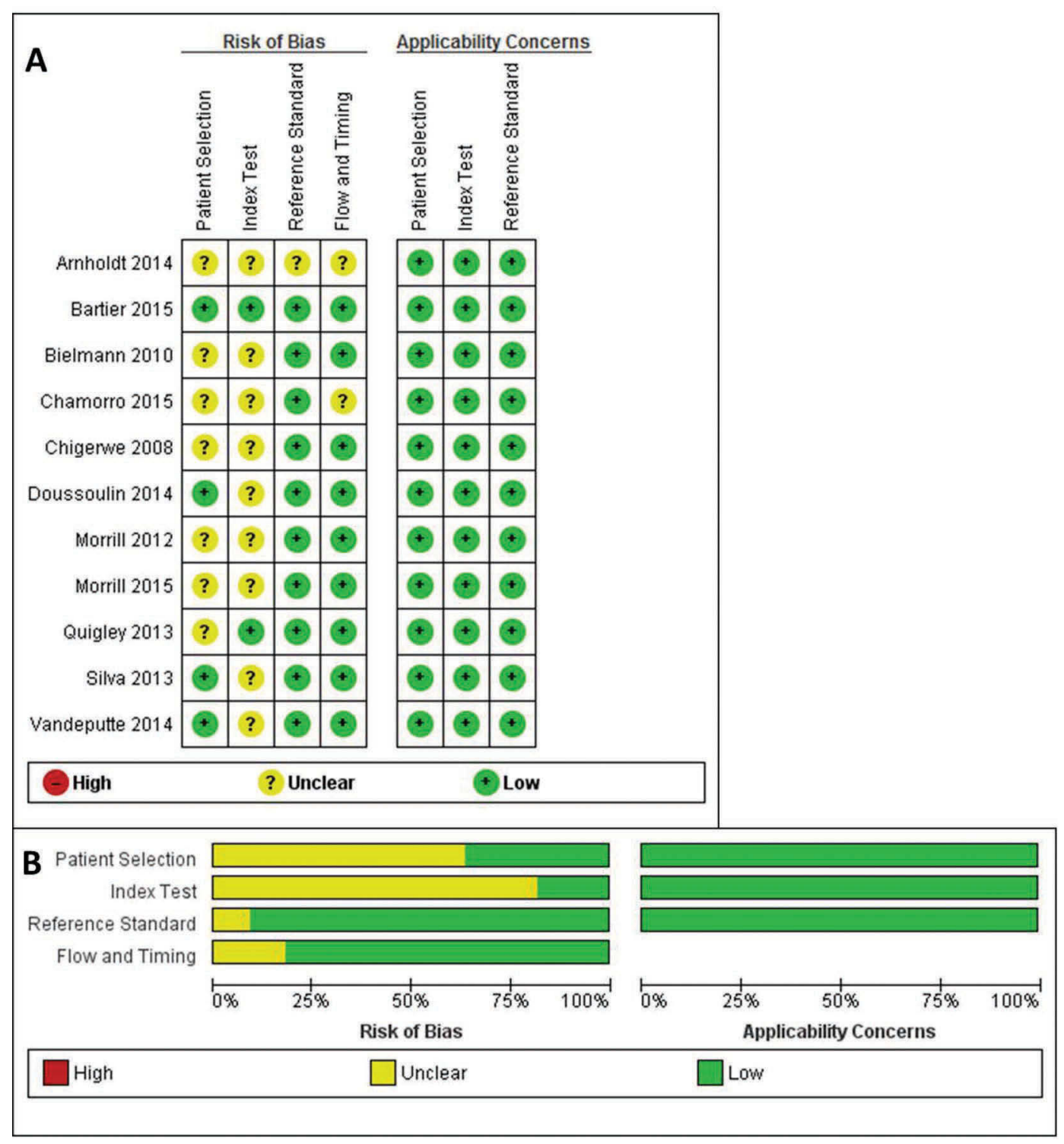

Figure 2. Risk of bias and applicability concerns for studies included in the systematic review based on quality of diagnostic accuracy studies (QUADAS-2). The individual (A) and summary (B) results are indicated concerning the risk of bias and applicability concerns. Color version available online.

reporting a systematic review of diagnostic test accuracy. Choosing a commonly used IgG concentration cut-point of $50 \mathrm{~g} / \mathrm{L}$ (determined with RID or NIRS) to define a "good colostrum" is debatable, because there is not clear evidence that this threshold definitely classifies the quality of colostrum. This approach would classify 2 colostrum samples with 49 or $51 \mathrm{~g} / \mathrm{L}$ of $\mathrm{IgG}$ as not-good and good, respectively, even though this small difference is not likely to be clinically relevant. The IgG concentration is only a part of assessment of colostrum quality. Other factors including live maternal cells, cytokines, growth factors, nutrient composition, and bacterial contamination also need to be taken into account when assessing the overall quality of colostrum delivered to the calf (Godden, 2008). Bayesian hierarchical models can be used to take into account the imperfection of the reference standard (Dendukuri et al., 2012) considering that the "true colostrum quality" is a latent variable that is assessed with an imperfect reference standard test (i.e., $50 \mathrm{~g} / \mathrm{L} \mathrm{IgG} \mathrm{cut-point).} \mathrm{We}$ did not choose this modeling strategy because there was not any prior information concerning the anticipated accuracy of the reference standard (for modeling information). We also concluded that assessing the accuracy 


\begin{tabular}{|c|c|c|c|c|c|c|c|c|}
\hline Study & TP & FP & FN & TN & Sensitivity (95\% Cl) & Specificity (95\% Cl) & Sensitivity (95\% Cl) & Specificity (95\% Cl) \\
\hline Arnholdt 2014 & 45 & 1 & 22 & 18 & $0.67[0.55,0.78]$ & $0.95[0.74,1.00]$ & $\rightarrow-$ & $\longrightarrow$ \\
\hline Bartier 2015 & 270 & 46 & 56 & 88 & $0.83[0.78,0.87]$ & $0.66[0.57,0.74]$ & - & \\
\hline Bielmann 2010 & 233 & 4 & 19 & 17 & $0.92[0.88,0.95]$ & $0.81[0.58,0.95]$ & $=$ & \\
\hline Chamorro 2015 & 946 & 60 & 267 & 317 & $0.78[0.76,0.80]$ & $0.84[0.80,0.88]$ & ש & \\
\hline Chigerwe 2008 & 91 & 14 & 26 & 41 & $0.78[0.69,0.85]$ & $0.75[0.61,0.85]$ & $\rightarrow$ & - \\
\hline Doussoulin 2014 & 72 & 3 & 10 & 15 & $0.88[0.79,0.94]$ & $0.83[0.59,0.96]$ & - & $\rightarrow-$ \\
\hline Morrill 2012 & 549 & 19 & 38 & 222 & $0.94[0.91,0.95]$ & $0.92[0.88,0.95]$ & - & 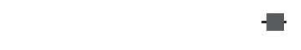 \\
\hline Morrill 2015 & 36 & 1 & 3 & 18 & $0.92[0.79,0.98]$ & $4,1.00]$ & $\longrightarrow$ & $\longrightarrow$ \\
\hline Quigley 2013 & 143 & 10 & 11 & 19 & $0.93[0.88,0.96]$ & 0.82 & $-\frac{1}{n^{2}}$ & 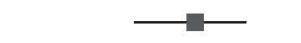 \\
\hline Silva 2013 & 86 & 5 & 5 & 9 & $8,0.98]$ & $5,0.87]$ & - 를 & - \\
\hline Vandeputte 2014 & 340 & 5 & 20 & 31 & $0.94[0.92,0.97]$ & $0.86[0.71,0.95]$ & 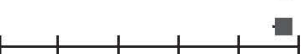 & $\rightarrow-$ \\
\hline
\end{tabular}

Figure 3. Forest plots of paired sensitivities and specificities of studies reporting the diagnostic accuracy of Brix refractometry to detect good quality colostrum $(\operatorname{IgG} \geq 50 \mathrm{~g} / \mathrm{L})$. TP $=$ true positive (Brix $\geq$ cut-off and $\operatorname{IgG} \geq 50 \mathrm{~g} / \mathrm{L}$ ); FP $=$ false positive (Brix $\geq$ cut-off and IgG $<50$ $\mathrm{g} / \mathrm{L}$ ); FN = false negative (Brix $<$ cut-off and IgG $\geq 50 \mathrm{~g} / \mathrm{L}$ ) $; \mathrm{TN}=$ true negative (Brix $<$ cut-off and $\mathrm{IgG}<50 \mathrm{~g} / \mathrm{L}$ ) $; \mathrm{sensitivity}=\mathrm{TP} /(\mathrm{TP}+$ $\mathrm{FN})$; specificity $=\mathrm{TN} /(\mathrm{TN}+\mathrm{FP})$.

of Brix for determining the $50 \mathrm{~g} / \mathrm{L}$ of $\mathrm{IgG}$ concentration cut-point was anticipated to be more relevant for application in the field.

Besides the estimation of specific Se/Sp values for different cut-points, which is the classical focus of diagnostic test accuracy review, we tried to translate

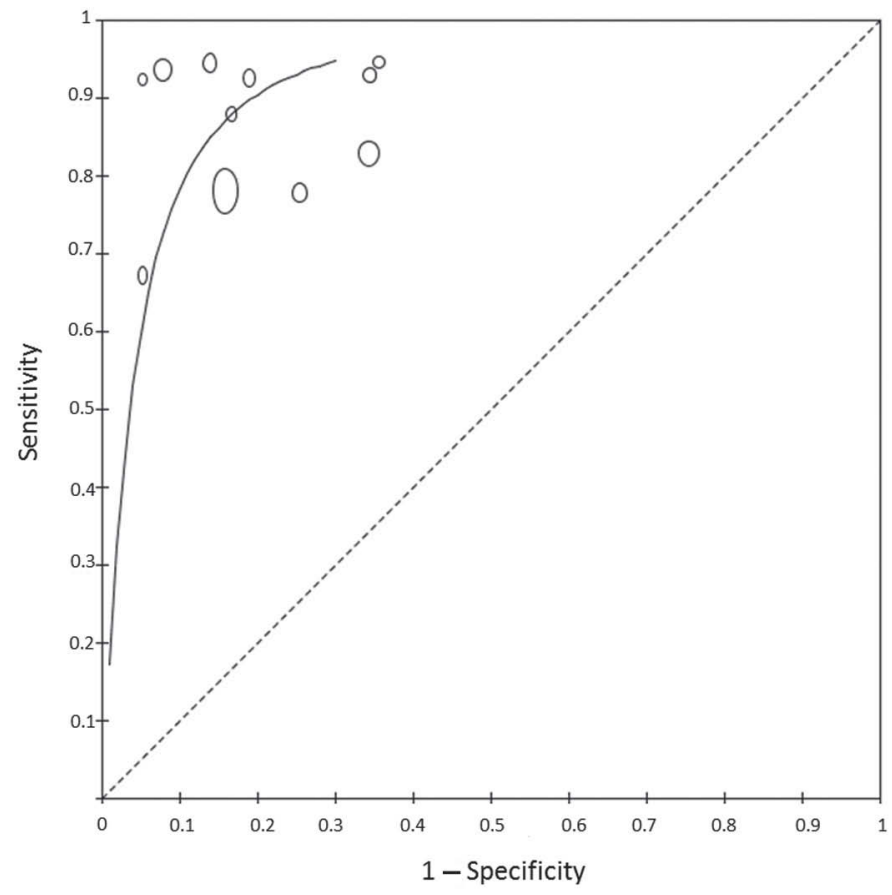

Figure 4. Hierarchical summary receiver operating characteristic (HSROC) curve of the diagnostic accuracy of the Brix refractometer to detect colostrum of good quality ( $\operatorname{IgG} \geq 50 \mathrm{~g} / \mathrm{L}$ ). The HSROC curve was obtained using the proposed accuracy parameters in every study. The size of the bubbles is proportional to the variance of sensitivity (y-axis) and specificity (x-axis) estimates. these findings to a more practical interpretation with stochastic modeling. The Se and Sp can be difficult to interpret in practical terms, so it is important to illustrate the main findings of the diagnostic test assessment (Leeflang et al., 2013; Zhelev et al., 2013). Therefore we used models to demonstrate the implications of our findings.

Our review demonstrated that a specific cut-point of $\geq 22 \%$ (which was the most commonly reported cutpoint) as a positive Brix refractometry result could be used as a reliable tool to identify good quality colostrum (compatible with good quality colostrum in $94.3 \%$ (5th-95th percentile: 90.7-96.9\%) of cases). However, we show that applying a threshold of $22 \%$ Brix would misclassify a considerable amount of good quality colostrum (which would possibly be discarded), especially when prevalence of good colostrum is high as in many reported studies. On the basis of the reported prevalence of colostrum samples with an IgG concentration $\geq 50 \mathrm{~g} / \mathrm{L}$ (which, on average, was close to $80 \%$ ), our meta-analysis demonstrated that there was still close to a $50 \%$ [47.2\% (5th-95th percentile: $35.5-62.3 \%$ )] chance that colostrum with a Brix result $<22 \%$ was good quality colostrum. Consequently, this indicates that using the same cut-point $(<22 \%)$ to define a negative test to determine if colostrum should be discarded can lead to a large amount of good colostrum being discarded. This threshold effect is frequently observed in diagnostic testing (Park et al., 2004; Leeflang et al., 2008). Depending on the context of application of Brix testing, the implications of this could differ.

In the current meta-analysis with stochastic modeling, using a lower Brix cut-point to discard poor quality colostrum appeared to be a more reasonable way to reject colostrum of poor quality. A colostrum sample 
$<18 \%$ Brix would only have 20 to $25 \%$ chance of being good quality colostrum. According to our current findings, a test result $<18$ and $\geq 22 \%$ on the Brix scale could be used advantageously to define poor colostrum and good colostrum, respectively. The samples between $\geq 18$ and $<22 \%$ Brix could be considered as suspect and supplementation with colostrum supplements or replacers would be recommended. Increasing the volume fed to the calf could also be an option for colostrum with these Brix values to feed at least $200 \mathrm{~g}$ of $\mathrm{IgG}$ per calf; however, the exact extra-volume needed cannot be precisely determined because it is difficult to predict the true IgG content of these samples. Several different regression equations have been previously published

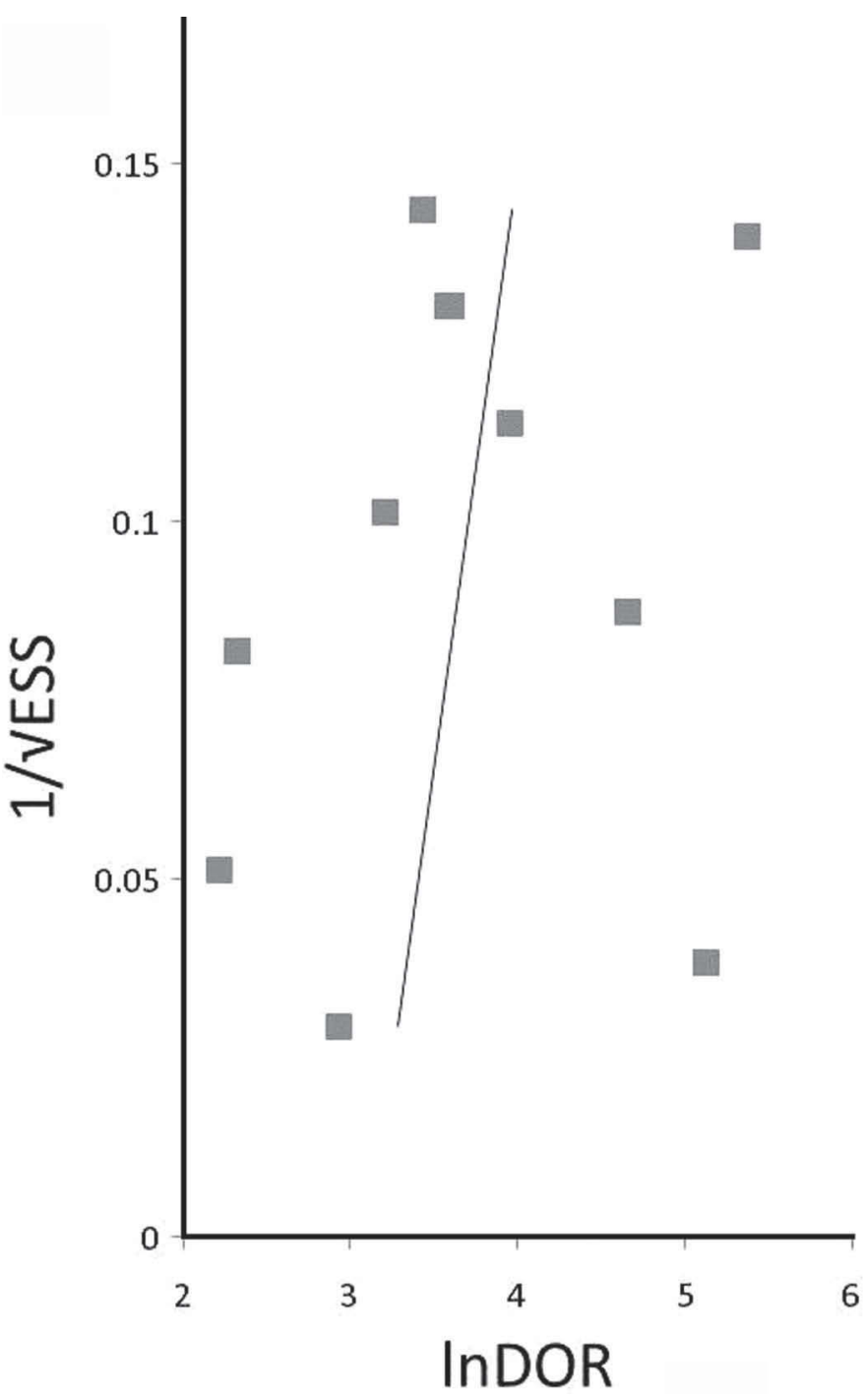

Figure 5. Deeks plot for assessing publication bias for the systematic review. The association between $\operatorname{lnDOR}$ and $1 / \sqrt{ }($ ESS $)$ was not significant $(P=0.48)$. lnDOR $=$ natural logarithm of the diagnostic odds ratio of the test; ESS = effective sample size.
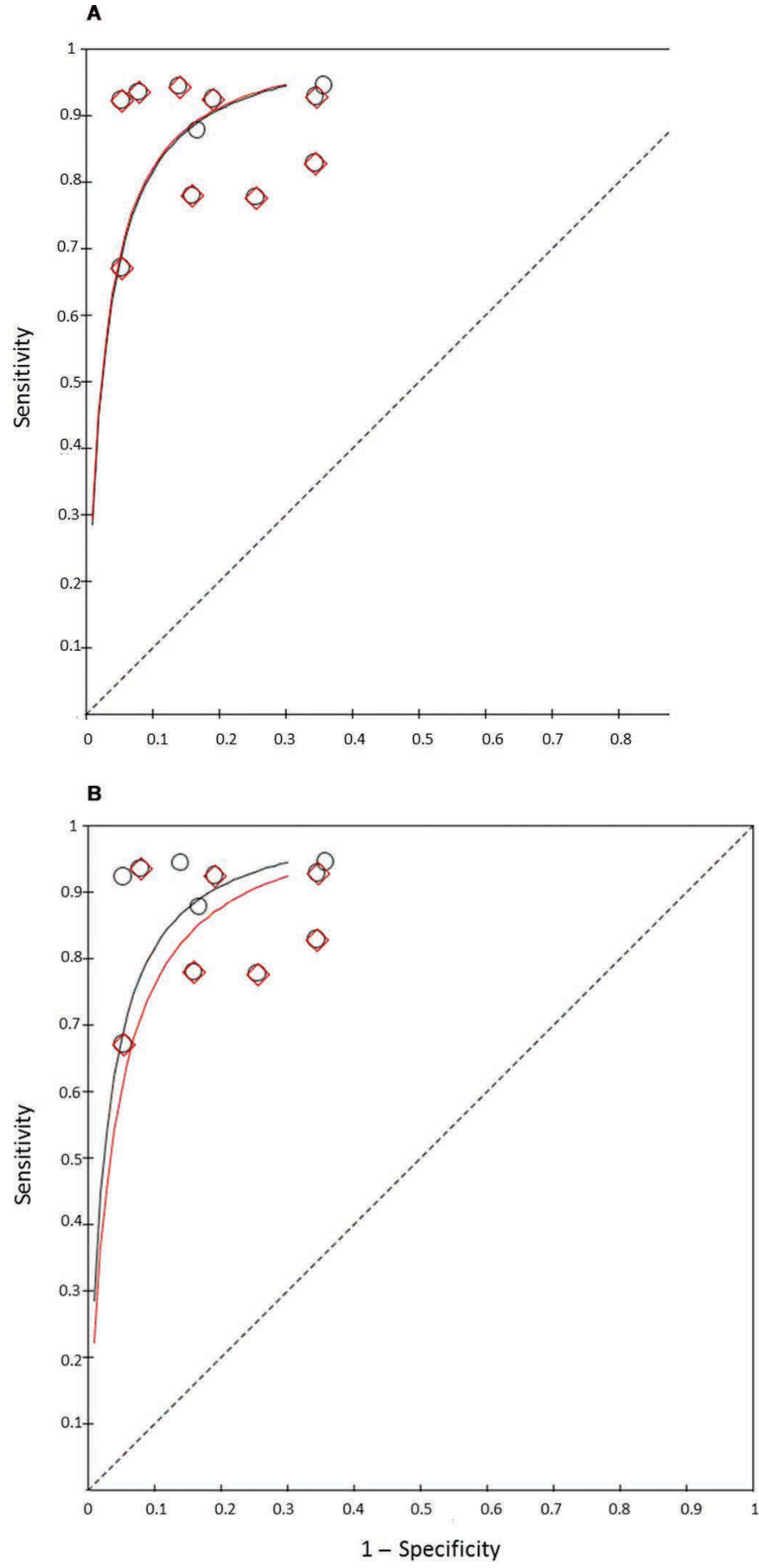

Figure 6. Sensitivity analysis and summary receiver operating characteristics (ROC) curves when removing studies assessing IgG concentration with near infrared spectrometry (A) and including a specific breed of cattle (Jersey or beef cows) (B). (A) Circle: all ROC estimates for every included study; diamond: ROC estimates for studies determining IgG concentration using near infrared spectrometry (NIRS vs. radial immunodiffusion; Silva, 2013; Doussoulin, 2014). (B) Circle: all ROC estimates for every included study; diamond: ROC estimates for studies determining IgG concentration using NIRS (Silva, 2013; Doussoulin, 2014) or studies using other cow breeds (Vandeputte et al., 2014; Morrill et al., 2015). Color version available online. 
Table 2. Specific accuracy estimates of prespecified cut-offs and effect of these estimates on posttest probability of good colostrum quality knowing the Brix result (below or above the cut-off) ${ }^{1}$

\begin{tabular}{lccc}
\hline Cut-off & $18\left(\mathrm{n}^{2}=6\right)$ & $21\left(\mathrm{n}^{2}=5\right)$ & $22\left(\mathrm{n}^{2}=8\right)$ \\
\hline Sensitivity (95\% CI) & $96.1(91.8-98.2)$ & $87.6(76.4-93.9)$ & $80.2(71.1-87.0)$ \\
Specificity (95\% CI) & $54.5(26.9-79.6)$ & $79.0(51.3-93.1)$ & $82.6(71.4-90.0)$ \\
LR+ (95\% CI) & $2.11(1.14-3.93)$ & $4.17(1.65-10.55)$ & $4.61(2.97-7.16)$ \\
LR- (95\% CI) & $0.072(0.046-0.112)$ & $0.157(0.096-0.257)$ & $0.240(0.177-0.324)$ \\
DOR (95\% CI) & $29.46(14.43-60.16)$ & $26.48(12.02-58.34)$ & $19.24(14.62-25.31)$ \\
$P_{\text {colostrum+| } \geq \text { cut-off }}$ & $88.7(82.0-93.9)$ & $93.5(88.0-97.1)$ & $94.3(90.7-96.9)$ \\
$P_{\text {colostrum+| < cut-off }}$ & $22.7(12.3-39.2)$ & $38.5(24.9-55.6)$ & $47.2(35.5-62.3)$ \\
\hline
\end{tabular}

${ }^{1} \mathrm{LR}+=$ likelihood ratio of a positive test (i.e., $\geq$ cut-off); LR $-=$ likelihood ratio of a negative test (i.e., $<$ cut-off); $\mathrm{DOR}=$ diagnostic odds ratio; $P_{\text {colostrum }+1} \geq$ cut-off $=$ probability that the colostrum will be good quality colostrum ( $\operatorname{IgG} \geq 50 \mathrm{~g} / \mathrm{L})$ knowing the Brix value is $\geq$ cut-off. The median, 5th and 95th percentile of the distribution obtained from 50,000 iterations of Monte Carlo simulation; $P_{\text {colostrum }+1<\text { cut-off }}=$ probability that the colostrum will be good quality colostrum ( $\operatorname{IgG} \geq 50 \mathrm{~g} / \mathrm{L}$ ) knowing the Brix value is $<$ cut-off. The median, 5 th and 95th percentile of the distribution obtained from 50,000 iterations of Monte Carlo simulation.

${ }^{2}$ Where $\mathrm{n}=$ number of studies where $2 \times 2$ table information was available for the specific cut-off.

correlating colostrum Brix percentage and IgG concentration (Quigley et al., 2013). However, their direct implementation on the farm level is difficult, and the accuracy of prediction has not been specifically validated. Using a cut-point approach is therefore more easily understood and easy to explain to the calf-care team using a coding system and decision tree for colostrum quality. For example, if the colostrum is of good quality ( $\geq 22 \%$ Brix), then feed $4 \mathrm{~L}$ of this colostrum as

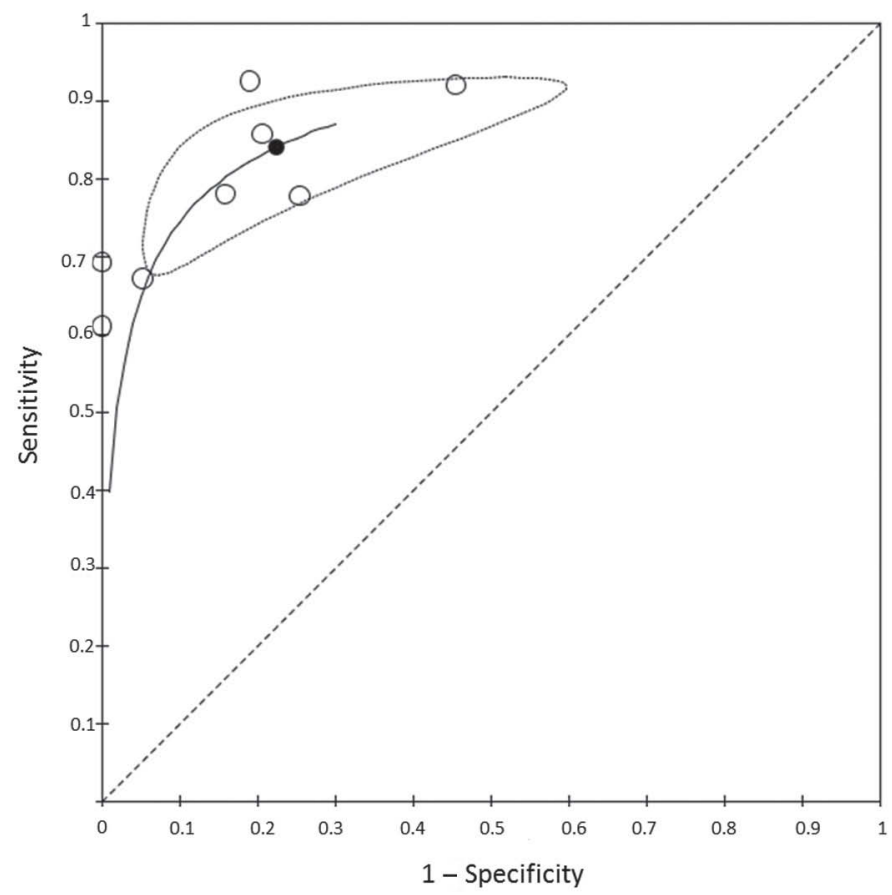

Figure 7. Hierarchical summary receiver operating characteristic curve (HSROC), summary point estimate, and $95 \%$ CI for Brix refractometry accuracy using a cut-point of $22 \%$ for determining good quality colostrum (IgG concentration $\geq 50 \mathrm{~g} / \mathrm{L}$ ). soon as possible for a Holstein calf. If the colostrum fails ( $<18 \%$ Brix), then do not use this sample but use frozen colostrum or other colostrum replacer, and last, if the colostrum is mid-range $(18 \% \leq$ Brix $<22 \%)$, then a mix of colostrum and colostrum replacer can be used.

These recommendations are based on the studies assessed in the present meta-analysis, which on average had high prevalence of good quality colostrum (median $77.8 \%$ ). What if, however, the prevalence of good quality colostrum was lower [i.e., closer to a previous US survey showing that up to $30 \%$ of colostrum had $\operatorname{IgG}$ levels <50 g/L (Morrill et al., 2012a)]? Using lower prevalence of good quality colostrum in our model (i.e., minimum: $60 \%$, mode: $70 \%$, maximum: $80 \%$ ) did not significantly change our findings with a probability of good colostrum at $91.3 \%$ for samples $\geq 22 \%$ and $15.4 \%$ for samples $<18 \%$ Brix (data not shown).

The assessment of the diagnostic accuracy of the Brix refractometer for colostrum quality determination using an evidence-based approach posed several challenges. Only $10 \%$ of the studies initially identified during reference search could be used for this systematic review. Some studies did not allow the determination of $2 \times 2$ tables accurately $(\mathrm{n}=3$, Figure 1$)$ and focused more on the association between the index test and reference standard in a continuous scale (e.g., linear regression and $R^{2}$ ) rather than presenting specific cutpoint information or only reporting Se and Sp without information on prevalence of good quality colostrum. For this reason it is important to continue gathering specific information on this topic as well as on several covariates that could potentially have an effect on test accuracy (e.g., breed, parity, pooled, or heat-treated colostrum, type of refractometer used, colostrum color, and so on) to refine the current findings. Reporting all information allowing the construction of $2 \times 2$ tables or any study limitation is important for future updates of 
this systematic review. Following the standards for reporting diagnostic accuracy study (STARD) developed in human medicine would help to standardize reporting (Bossuyt et al., 2003).

The external validity of the studies raised no applicability concerns. The selected studies were fully applicable to our review question. For this reason, we think the results of our review can be applicable in many farms. The average quality of reporting was good despite unclear risk of bias for patient selection (due to the absence of reporting the details of sampling strategy) and index test. The specification of sampling strategy is a key concern in diagnostic test accuracy review because it is important to know if all (or a random sample of) eligible patients or samples had been submitted to the index test and the reference standard. In the specific context of this review we could not exactly know in several studies if some samples with specific characteristics (bloody or watery sample) had been withdrawn and therefore we could not be $100 \%$ sure that this type of study bias was avoided.

Because it was not precisely indicated if the Brix test was interpreted blind to the reference standard results or if the cut-point was data-driven, over-optimistic as- sessment of test accuracy was possible (Leeflang et al., 2008). Again, this is a commonly mentioned problem in systematic reviews when it is not possible to give a valid judgement on a specific item because the information cannot be retrieved from the manuscript (Whiting et al., 2011). We could not assess the effect of some covariates due to the small number of studies where these specific covariates were taken into account (e.g., cow parity, breed, type of refractometer used). It is well known that test accuracy can be affected by specific sample characteristics and ignoring these could lead to misinterpretations of some test results (Macaskill et al., 2010). As an example, we could not assess the robustness of the cut-points proposed in specific breeds or in specific parity groups because of the small number of studies mentioning these specific characteristics.

\section{CONCLUSIONS}

This systematic review meta-analysis showed that Brix refractometry is an accurate test for diagnosing good quality colostrum. Based on our results and stochastic modeling, a Brix result $\geq 22 \%$ could be used to define good quality colostrum and could help decide

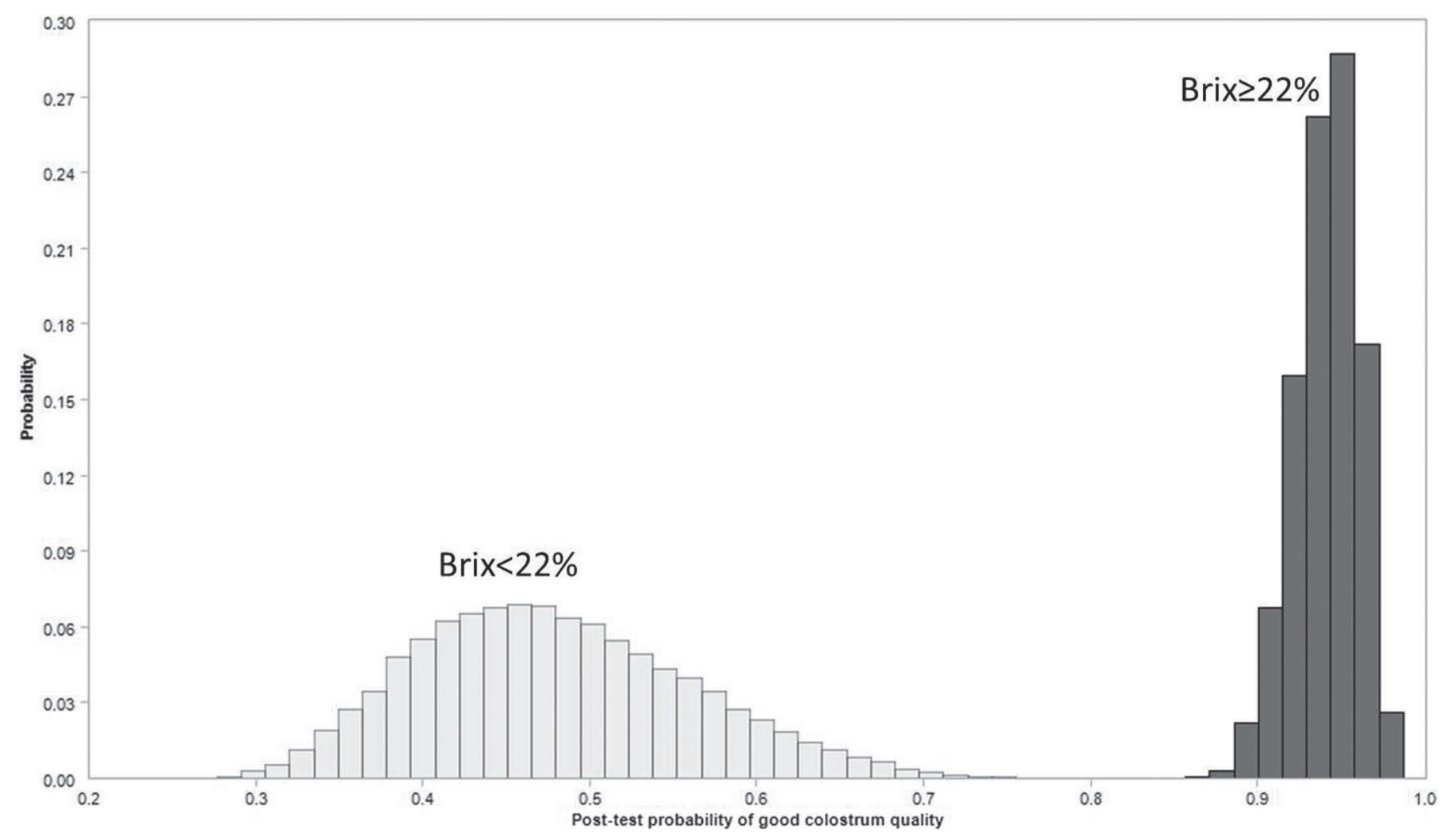

Figure 8. Model simulation of posttest probability of having a good quality colostrum based on a positive $(\geq 22 \%)$ or negative $(<22 \%)$ Brix results. The model was based on 50,000 iterations using a Monte Carlo simulation. The y-axis indicates probability density. The posttest probability was obtained based on the likelihood ratio of a positive (dark gray) or negative (light gray) Brix result and Bayes theorem. 
which colostrum to keep when using a frozen colostrum bank. A Brix value $<18 \%$ is compatible with low quality colostrum and specific action should be taken if using this sample for calf feeding (such as using colostrum replacer, higher volumes, or colostrum supplements). The colostrum with $18 \leq$ Brix $<22 \%$ can be considered as suspect, and adding frozen colostrum or colostrum supplement is recommended.

\section{REFERENCES}

Arnholdt, T., D. Klein-Joebstl, K. Rychli, S. Borchardt, M. Iwersen, and M. Drillich. 2014. Estimation of colostrum quality in the field by Brix refractometry. Page 9 in Proceedings Reproduction in Domestic Animals. Wiley Blackwell, Hoboken, NJ.

Bartier, A. L., M. C. Windeyer, and L. Doepel. 2015. Evaluation of on-farm tools for colostrum quality measurement. J. Dairy Sci. 98:1878-1884.

Baumrucker, C. R., and R. M. Bruckmaier. 2014. Colostrogenesis: IgG1 transcytosis mechanisms. J. Mamm. Gland Biol. Neoplasia 19:103-117.

Baumrucker, C. R., A. M. Burkett, A. L. Magliaro-Macrina, and C. D. Dechow. 2010. Colostrogenesis: Mass transfer of immunoglobulin $\mathrm{G}_{1}$ into colostrum. J. Dairy Sci. 93:3031-3038.

Bielmann, V., J. Gillan, N. R. Perkins, A. L. Skidmore, S. Godden, and K. E. Leslie. 2010. An evaluation of Brix refractometry instruments for measurement of colostrum quality in dairy cattle. J. Dairy Sci. 93:3713-3721.

Bossuyt, P. M., J. B. Reitsma, D. E. Bruns, C. A. Gatsonis, P. P Glasziou, L. M. Irwig, J. G. Lijmer, D. Moher, D. Rennie, and H. C. de Vet. 2003. Towards complete and accurate reporting of studies of diagnostic accuracy: The STARD Initiative. Ann. Intern. Med. 138:40-44.

Caraguel, C. G., and R. Vanderstichel. 2013. The two-step Fagan's nomogram: Ad hoc interpretation of a diagnostic test result without calculation. Evid. Based Med. 18:125-128.

Chamorro, M. F., R. Sargent, D. M. Haines, and J. Lombard. 2015. Evaluation of the Brix refractometer for measuring colostral and serum IgG concentrations. J. Dairy Sci. 98(S2):236.

Chigerwe, M., and J. V. Hagey. 2014. Refractometer assessment of colostral and serum IgG and milk total solids concentrations in dairy cattle. BMC Vet. Res. 10:178.

Chigerwe, M., J. W. Tyler, J. R. Middleton, J. N. Spain, J. S. Dill, and B. J. Steevens. 2008. Comparison of four methods to assess colostral IgG concentration in dairy cows. J. Am. Vet. Med. Assoc. 233:761-766.

Conneely, M., D. P. Berry, R. Sayers, J. P. Murphy, I. Lorenz, M. L. Doherty, and E. Kennedy. 2013. Factors associated with the concentration of immunoglobulin $\mathrm{G}$ in the colostrum of dairy cows. Animal 7:1824-1832.

Deeks, J. J., P. Macaskill, and L. Irwig. 2005. The performance of tests of publication bias and other sample size effects in systematic reviews of diagnostic test accuracy was assessed. J. Clin. Epidemiol. 58:882-893.

Deelen, S. M., T. L. Ollivett, D. M. Haines, and K. E. Leslie. 2014. Evaluation of a Brix refractometer to estimate serum immunoglobulin G concentration in neonatal dairy calves. J. Dairy Sci. 97:3838-3844.

Dendukuri, N., I. Schiller, L. Joseph, and M. Pai. 2012. Bayesian metaanalysis of the accuracy of a test for tuberculous pleuritis in the absence of a gold standard reference. Biometrics 68:1285-1293.

Doussoulin, J. 2014. Determinación de la calidad de calostro mediante la calibración de un refractómetro Brix en vacas Holstein a pastoreo. Thesis. Facultad de Ciencias Agrarias, Universidad Austral de Chile, Valdivia, Chile.

Fleenor, W. A., and G. H. Stott. 1981. Single radial immunodiffusion analysis for quantitation of colostral immunoglobulin concentration. J. Dairy Sci. 64:740-747.
Gelsinger, S. L., C. M. Jones, and A. J. Heinrichs. 2015. Effect of colostrum heat treatment and bacterial population on immunoglobulin G absorption and health of neonatal calves. J. Dairy Sci. 98:4640-4645.

Godden, S. 2008. Colostrum management for dairy calves. Vet. Clin. North Am. Food Anim. Pract. 24:19-39.

Gross, J. J., E. C. Kessler, and R. M. Bruckmaier. 2014. Colour measurement of colostrum for estimation of colostral IgG and colostrum composition in dairy cows. J. Dairy Res. 81:440-444.

Leeflang, M. M. 2014. Systematic reviews and meta-analyses of diagnostic test accuracy. Clin. Microbiol. Infect. 20:105-113.

Leeflang, M. M., J. J. Deeks, Y. Takwoingi, and P. Macaskill. 2013. Cochrane diagnostic test accuracy reviews. Syst. Rev. 2:82.

Leeflang, M. M., K. G. Moons, J. B. Reitsma, and A. H. Zwinderman. 2008. Bias in sensitivity and specificity caused by data-driven selection of optimal cutoff values: Mechanisms, magnitude, and solutions. Clin. Chem. 54:729-737.

Lehman, D., H. Groenendaal, and G. Nolder. 2011. Practical Spreadsheet Risk Modeling for Management. CRC Press, Boca Raton, FL.

Macaskill, P., C. Gatsonis, J. Deeks, R. Harbord, and Y. Takwoingi. 2010. Chapter 10: Analysing and presenting results. Cochrane handbook for systematic reviews of diagnostic test accuracy version 1(0). Accessed Jun. 27, 2016. http://methods.cochrane.org/sdt/ sites/methods.cochrane.org.sdt/files/uploads/Chapter\%2010\%20 $-\% 20$ Version\%201.0.pdf.

McGuirk, S. M., and M. Collins. 2004. Managing the production, storage, and delivery of colostrum. Vet. Clin. North Am. Food Anim. Pract. 20:593-603.

Morrill, K. M., E. Conrad, A. Lago, J. Campbell, J. Quigley, and H. Tyler. 2012a. Nationwide evaluation of quality and composition of colostrum on dairy farms in the United States. J. Dairy Sci. 95:3997-4005.

Morrill, K. M., E. Conrad, J. Polo, A. Lago, J. Campbell, J. Quigley, and H. Tyler. 2012b. Estimate of colostral immunoglobulin G concentration using refractometry without or with caprylic acid fractionation. J. Dairy Sci. 95:3987-3996.

Morrill, K. M., K. E. Robertson, M. M. Spring, A. L. Robinson, and H. D. Tyler. 2015. Validating a refractometer to evaluate immunoglobulin G concentration in Jersey colostrum and the effect of multiple freeze-thaw cycles on evaluating colostrum quality. J. Dairy Sci. 98:595-601.

Osaka, I., Y. Matsui, and F. Terada. 2014. Effect of the mass of immunoglobulin (Ig)G intake and age at first colostrum feeding on serum IgG concentration in Holstein calves. J. Dairy Sci. 97:66086612.

Park, S. H., J. M. Goo, and C. H. Jo. 2004. Receiver operating characteristic (ROC) curve: Practical review for radiologists. Korean J. Radiol. 5:11-18.

Quigley, J. D., A. Lago, C. Chapman, P. Erickson, and J. Polo. 2013. Evaluation of the Brix refractometer to estimate immunoglobulin G concentration in bovine colostrum. J. Dairy Sci. 96:1148-1155.

Rivero, M. J., X. Valderrama, D. Haines, and D. Alomar. 2012. Prediction of immunoglobulin $\mathrm{G}$ content in bovine colostrum by nearinfrared spectroscopy. J. Dairy Sci. 95:1410-1418.

Rutjes, A. W., J. B. Reitsma, J. P. Vandenbroucke, A. S. Glas, and P. M. Bossuyt. 2005. Case-control and two-gate designs in diagnostic accuracy studies. Clin. Chem. 51:1335-1341.

Rutter, C. M., and C. A. Gatsonis. 2001. A hierarchical regression approach to meta-analysis of diagnostic test accuracy evaluations. Stat. Med. 20:2865-2884.

Sangild, P. T. 2003. Uptake of colostral immunoglobulins by the compromised newborn farm animal. Acta Vet. Scand. Suppl. 98:105122

Sargeant, J. M., and A. M. O'Connor. 2014. Introduction to systematic reviews in animal agriculture and veterinary medicine. Zoonoses Public Health 61(Suppl. 1):3-9.

Silva, S. 2013. Calibración de refractómetro Brix para la determinación del contenido de Inmunoglobulina $\mathrm{G}$ en calostro bovino. Thesis. Facultad de Ciencias Agrarias, Universidad Austral de Chile, Valdivia, Chile. 
Son, H. S., Y. S. Hong, W. M. Park, M. A. Yu, and C. H. Lee. 2009. A novel approach for estimating sugar and alcohol concentrations in wines using refractometer and hydrometer. J. Food Sci. 74:C106C111.

Stelwagen, K., E. Carpenter, B. Haigh, A. Hodgkinson, and T. T. Wheeler. 2009. Immune components of bovine colostrum and milk. J. Anim. Sci. 87(Suppl.):3-9.

Stott, G. H., D. B. Marx, B. E. Menefee, and G. T. Nightengale. 1979 Colostral immunoglobulin transfer in calves I. Period of absorption. J. Dairy Sci. 62:1632-1638.

Takwoingi, Y., and J. Deeks. 2008. METADAS: A SAS macro for meta-analysis of diagnostic accuracy studies. Page 24 in Proc. Methods for Evaluating Medical Tests Symposium. Department of Public Health, Epidemiology and Biostatistics, University of Birmingham, Birmingham, UK.

Tyler, H., and H. Ramsey. 1991. Hypoxia in neonatal calves: Effect on intestinal transport of immunoglobulins. J. Dairy Sci. 74:19531956. van Enst, W. A., E. Ochodo, R. J. Scholten, L. Hooft, and M. M. Leeflang. 2014. Investigation of publication bias in meta-analyses of diagnostic test accuracy: A meta-epidemiological study. BMC Med. Res. Methodol. 14:70.

Vandeputte, S., J. Detilleux, and F. Rollin. 2014. Investigation of colostrum quality in beef cattle by radial immunodiffusion and brix refractometry. Vet. Rec. 175:353.

Vasseur, E., F. Borderas, R. I. Cue, D. Lefebvre, D. Pellerin, J. Rushen, K. M. Wade, and A. M. de Passille. 2010. A survey of dairy calf management practices in Canada that affect animal welfare. J. Dairy Sci. 93:1307-1315.

Whiting, P. F., A. W. Rutjes, M. E. Westwood, S. Mallett, J. J. Deeks, J. B. Reitsma, M. M. Leeflang, J. A. Sterne, and P. M. Bossuyt. 2011. QUADAS-2: A revised tool for the quality assessment of diagnostic accuracy studies. Ann. Intern. Med. 155:529-536.

Zhelev, Z., R. Garside, and C. Hyde. 2013. A qualitative study into the difficulties experienced by healthcare decision makers when reading a Cochrane diagnostic test accuracy review. Syst. Rev. 2:32. 